\title{
Comparison between Active power filter with selective control and conventional control for harmonic in photovoltaic systems
}

\author{
J. Osorio ${ }^{1}$, J. Montaña ${ }^{2}$ \\ ${ }^{1}$ Master Student of Department of Electrical and Electronic Engineering \\ Universidad del Norte \\ Barranquilla (Colombia) \\ Phone/Fax number:+00575 3509270, e-mail: jhoinero@uninorte.edu.co \\ ${ }^{2}$ Professor of Department of Electrical and Electronic Engineering \\ Universidad del Norte \\ Barranquilla (Colombia) \\ Phone/Fax number:+00575 3509270, e-mail: johnym@uninorte.edu.co
}

\begin{abstract}
In order to to improve the power quality from energy generated by the photovoltaic systems connected to the network, this paper presents the results of two types of filters, first the active power filter with conventional control scheme and second, active power filter with a selective control scheme in order to reduce the harmonic content injected by the inverter in a photovoltaic system. The results are presented and compared by means of harmonic distortion (individual and THD). This is a novel proposal, since studies before to this, always have been interested in the compensation of harmonics arising from nonlinear loads but rarely from a voltage source, as in this case.
\end{abstract}

\section{Key words}

Active power filters (APFs), power quality, harmonics current, photovoltaic inverter, selective control

\section{Introduction}

Nowadays the use of generation of clean and renewable energy, as is the case of photovoltaic systems, has been gradually becoming a viable alternative within the diagrams of distributed generation. The use of these clean energies is becoming a common practice in the world, with an annual growth rate of $16 \%$ [7].

To convert solar radiation into electrical energy is necessary the use a system composed of photovoltaic components. For systems connected to the network, the components are: photovoltaic field, photovoltaic inverter, protection devices and the energy meter. The photovoltaic inverter generates and injects harmonic content to the network, due to its switching during the conversion process of direct current (DC) to alternating current (AC) [11], being able to produce a negative impact on it, as well as in the final users. The previous thing is not present in other types of generation systems because they do not inject harmonic content to the network during the generation process.
The motivation of this work is to improve the power quality from the photovoltaic systems connected to the network, compensating the harmonic content injected by the photovoltaic inverter using active power filters (APFs). Two types of filters are going to be considered, conventional control scheme and selective control scheme. That is an innovative aspect within the literature, since studies prior to this, always have been interested in the compensation of harmonics arising from non-linear loads but rarely from a voltage source, as in this case.

The active filters are designed to take into account the operating characteristics and harmonic content of the photovoltaic inverter and, therefore, it adapts by itself to the dynamic operating conditions of the photovoltaic system, due to the stochastic nature of the solar radiation. When a conventional control is used, the filter controls the entire harmonics as a total, whereas the selective control eliminates the harmonics individually by means of selective regulators that treat every harmonic separately. The second one can control the harmonics more efficiently as is shown in this paper, due to the selective regulators improve the response of the filter because the filter is tuned to the interest frequencies (for example harmonics 3, 5, 7 and 9).

\section{Harmonics current generated by photo- voltaic systems}

The photovoltaic systems connected to the network are able to generate harmonic currents during the conversion process from DC to AC. This conversion process happens across the inverters which are composed of power electronic devices that constitute a current source that inject harmonics to the network [2].

The harmonic current generated by the photovoltaic systems is related to the performance of the inverter 
under different loading conditions. Consequently, the harmonic distortion generated by the photovoltaic systems depends strongly from the generated power, in such a way that when this power is low, the harmonic distortion is high; on the other hand, the harmonic distortion is low. This behavior is appreciated clearly in Figure $1^{1}$, which shows the change of the total harmonic distortion of current $\left(\mathrm{THD}_{\mathrm{i}}\right)$ of a photovoltaic generator ${ }^{2}$ in regard to the generation rate $P_{G} / P_{n}$, where $P_{G}$ is the generated power by the photovoltaic system and $P_{n}$ is the nominal power of the photovoltaic generator [2].

The analysis of the harmonic distortion caused by the photovoltaic system at the point of common coupling (PCC) is, at first, similar to the analysis that is realized to determine the harmonic content caused in an isolated system of interconnected inverters. Nevertheless, also takes into account some peculiarities as the influence of climate variables as: the temperature, solar radiation and shadow effect. These variables impact significantly on the current and voltage waveform and establish the operating conditions and generation [2].

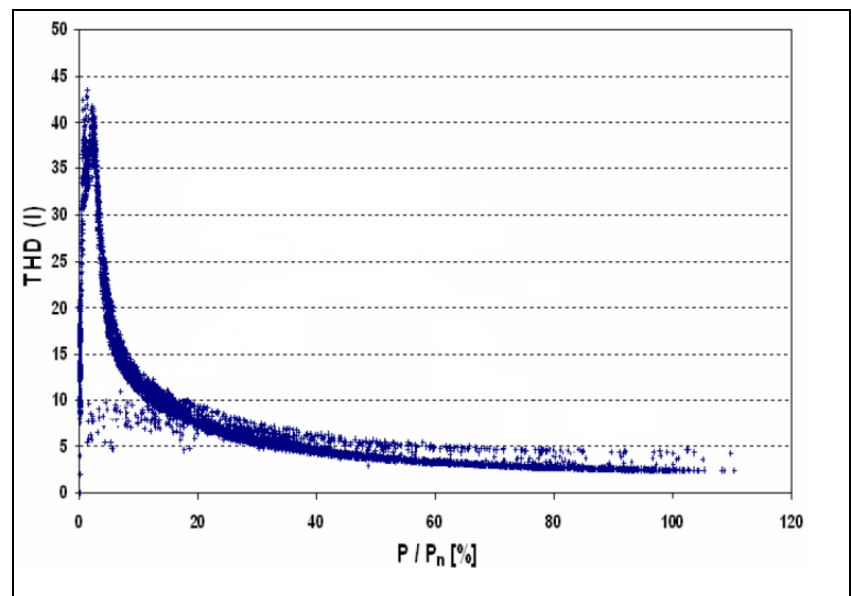

Fig. 1. Total harmonic distortion of a photovoltaic inverter $\left(\mathrm{P}_{\mathrm{DC} / \mathrm{AC}}=5 \mathrm{~kW}\right)$ for different loading conditions ${ }^{1}$

All types of photovoltaic inverter can be characterized by a similar dependency between the harmonic current injected into the network and the generated power [11]. Figure 2 relates the $\mathrm{THD}_{\mathrm{i}}$ and the generated power of an inverter of $5 \mathrm{~kW}$ throughout one summer day. During the ignition and shutdown of the generator, in the dawn and at nightfall respectively, the $\mathrm{THD}_{\mathrm{i}}$ for about 5 hours is higher than during the rest of the day.

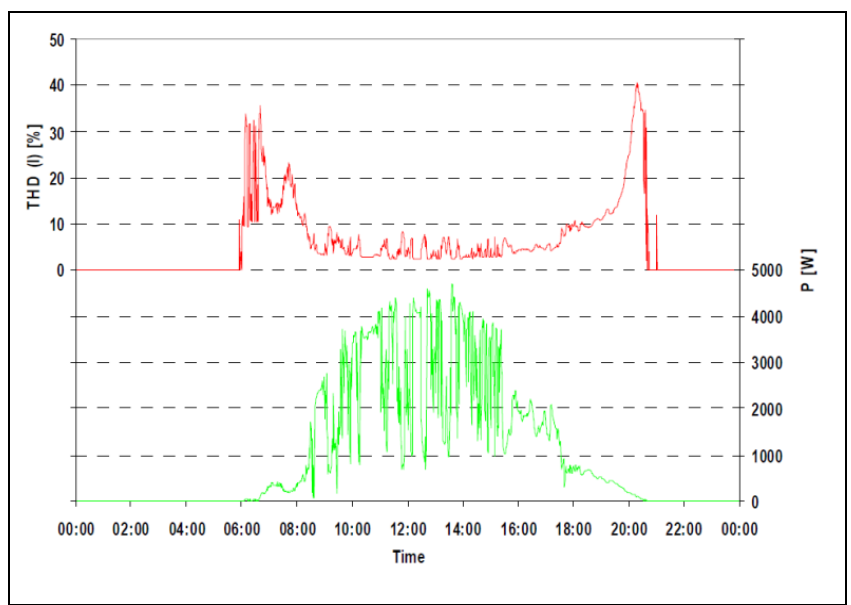

Fig. 2. THD $_{\mathrm{i}}$ (upper curve) and generated power (lower curve) of the photovoltaic inverter $\left(\mathrm{P}_{\mathrm{DC} / \mathrm{AC}}=5 \mathrm{~kW}\right)^{1}$

The individual current harmonics have a behavior similar to the $\mathrm{THD}_{\mathrm{i}}$, especially the harmonic of the lowest order. Figure 3 shows the relative values of the odd harmonics of current for different loading conditions in the inverter of $5 \mathrm{~kW}$. The even harmonics are negligible and they will not be considered, because their magnitudes are very low. Whereas, the harmonics of order 3, 5, 7 and 9 will be studied by special care, because they are the highest generated by the inverter.

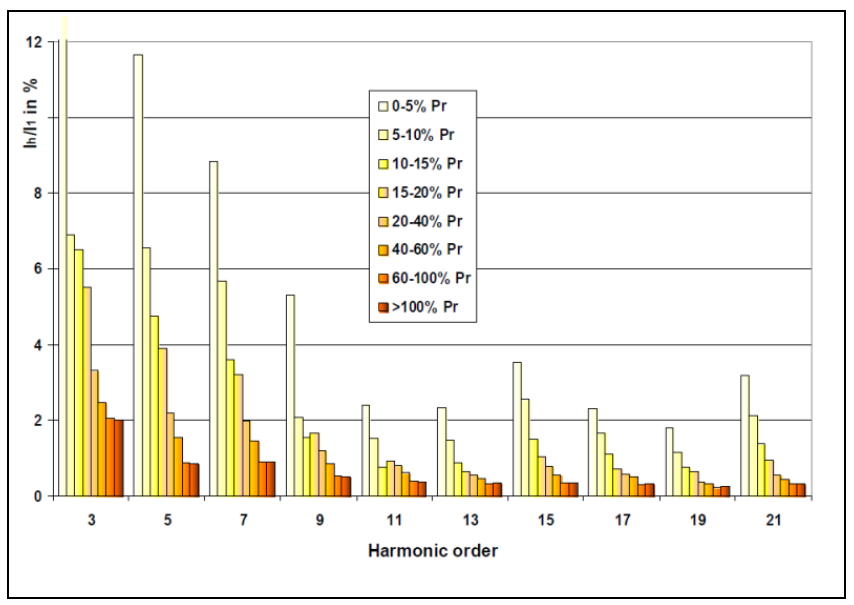

Fig. 3. Harmonic currents of the photovoltaic inverter $\left(\mathrm{P}_{\mathrm{DC} / \mathrm{AC}}=5\right.$ $\mathrm{kW}$ ) for different loading conditions ${ }^{1}$

\section{Control of shunt active power filter}

Figure 4 shows the scheme of a shunt APF connected to the PCC. At the same point, there are a linear load and the photovoltaic system, which makes circulate harmonic currents to the main electrical supply. The main goal of the APF control is to compensate the harmonic currents that the photovoltaic system injects to the network in order to avoid that these harmonics travel to the rest of the electrical system, that is to say, to achieve that the harmonic current is zero in the network. 


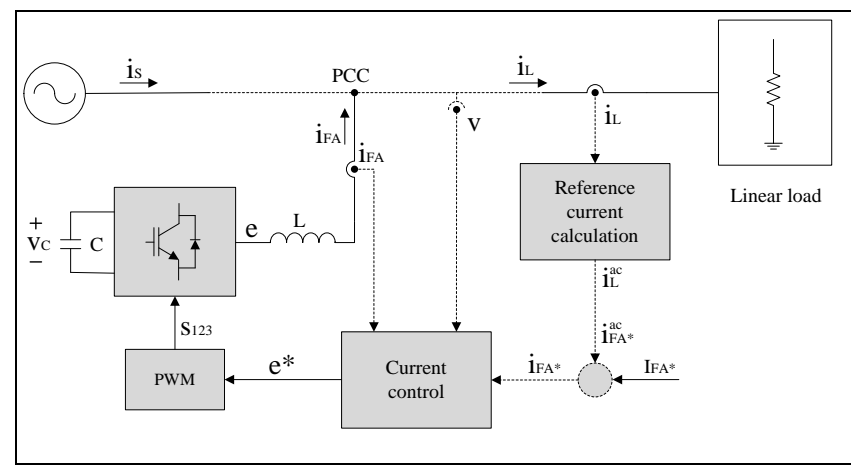

Fig. 4. Schematic diagram of the simulation with linear load

The control system of the shunt APF was divided in two parts: internal control and selective control. The internal control of the shunt active filter has the goal of controlling the output current and, simultaneously, the voltage of the condensers of the DC stage. Whereas, selective control has the task of improving the response of the shunt active filter for certain harmonics of interest [9].

\section{A. Internal control model of APF3.}

The dynamic equations of the shunt APF are divided into: the equations of network connection, the equations of power exchange and the equations of DC circuit. With the intention to make easier the control techniques, the Park's transformation is used to change the three-phase reference system (in axis $a, b$ and $c$ ), of the dynamic models of the shunt APF, to another orthogonal and equivalent reference system in axis $0, d$ and $q$ [8].

Applying the transformation to the dynamic equations of the shunt APF, is obtained:

- Equations of the circuit of network connection [9]:

$$
\begin{aligned}
& e_{d}-v_{d}=R i_{F A d}-L \omega_{1} i_{q}+L \frac{d i_{F A d}}{d t} \\
& e_{q}-v_{q}=R i_{F A q}+L \omega_{1} i_{d}+L \frac{d i_{F A q}}{d t}
\end{aligned}
$$

where $R$ and $L$ are the resistance and inductance of each coil connected to the network. The subscripts $d$ and $q$ indicate that they are a component in axis $d$ and $q$, respectively.

- $\quad$ Equations of power exchange [9]:

$$
\begin{aligned}
& p=v_{d} i_{F A d}+v_{q} i_{F A q} \\
& q=v_{d} i_{F A q}+v_{q} i_{F A d}
\end{aligned}
$$

where $p$ and $q$ are the instantaneous active and reactive power that the shunt APF injects into the system.

- $\quad$ Equation of the DC circuit [9]:

$$
\frac{1}{2} C \frac{d v_{C}^{2}}{d t}=-p
$$

where $C$ is the total capacitance of the condensers and $p$ is the power that goes out of the condensers. Equation (5) is linear, if it is taken $v_{C}^{2}$ as a variable of state. In this case, there are negligible the losses of the coil connection to the network. Consequently, (3) coincides with $p$ of the DC circuit.

Normally, the control system of the shunt APF is implemented in a digital platform. Consequently, the equations of the connecting circuit to the network (1) and (2) have to be discretized with a transformation (for a retainer of order zero), as it was made in [4] and [6]. In state variables the connection system is represented by:

$$
\begin{aligned}
& {\left[\begin{array}{c}
i_{F A d}(k+1) \\
i_{F A q}(k+1)
\end{array}\right]=\underbrace{\left[\begin{array}{cc}
\phi_{1} & \phi_{2} \\
-\phi_{2} & \phi_{1}
\end{array}\right]}_{\Phi} \underbrace{\left[\begin{array}{c}
i_{F A d}(k) \\
i_{F A q}(k)
\end{array}\right]}_{i_{F A d q}(k)}} \\
& +\underbrace{\left[\begin{array}{cc}
\gamma_{1} & \gamma_{2} \\
-\gamma_{2} & \gamma_{1}
\end{array}\right]}_{\Gamma} \underbrace{\left[\begin{array}{c}
e_{d}(k)-v_{d}(k) \\
e_{q}(k)-v_{q}(k)
\end{array}\right]}_{\Delta e_{d q}(k)}
\end{aligned}
$$

where $\boldsymbol{\Phi}$ and $\boldsymbol{\Gamma}$ correspond to the state matrixes at discrete time.

\section{B. Selective Control for APF3.}

The selective control is a technique of linear control that is based on Internal Model Principle (IMP) (Francis \& Wonham, 1975). This technique establishes that to follow a waveform without error it is necessary to include the model of that in the regulator of the control system. Therefore, to follow a sinusoidal waveform without error it is possible to include in the model this expression:

$$
S_{h}(s)=K_{h} \frac{s}{s^{2}+\left(2 h \pi / T_{p}\right)^{2}}
$$

where $K_{h}$ is the gain of the transfer function, $T_{p}$ is the period of the signal and $h$ is the harmonic component.

It is necessary to emphasize that to eliminate several harmonic frequencies it is necessary to include inside the control loop so many transfer functions (7) in series or in parallel as harmonic to be eliminated.

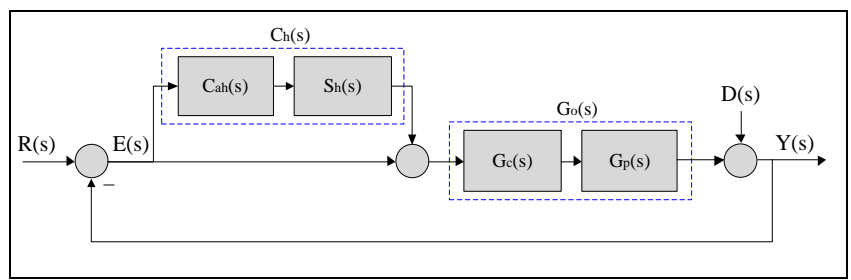

Fig. 5. Selective controller structure in continuous-time

Figure 5 shows the diagram of blocks of a selective regulator in continuous time. In this case, the selective regulator is added to a plant, $G_{p}(s)$, controlled by a conventional regulator, $G_{c}(s)$. The selective regulator has the form [5]:

${ }^{3}$ Taken from [9]. 


$$
C_{h}(s)=\underbrace{\frac{1+\left(\alpha_{c} / \omega_{h}\right) s}{1+f\left(\alpha_{c} / \omega_{h}\right) s}}_{C_{a h}(s)} \cdot \underbrace{\frac{K_{h} \omega_{h} s}{s^{2}+\omega_{h}^{2}}}_{S_{h}(s)}
$$

where $S_{h}(s)$ is the model of the sinusoidal waveform and $C_{a h}(s)$ is a leading (or lagging) compensation network that is added to stabilize the system.

It is necessary to clarify that the control algorithms used in this work for the implementation of the active filter with selective compensation have been designed and proved, for non-linear loads, in [10] and, therefore, they have been modified to adapt them to the characteristics of harmonic content that appear in the photovoltaic systems, where the harmonics of order $3,5,7$ and 9 predominate [11]. The main function of these control algorithms is to eliminate each current harmonic by means of selective regulators, which is tuned to certain frequencies of interest reducing the monitoring error in the control system.

There have been designed two selective regulators $C_{4}(s)$ and $C_{8}(s)$, for the harmonic 4 and 8 in components $d q 0$, respectively, which are equivalent to the harmonic $3,5,7$ and 9 in the three-phase system $a b c$.

\section{Experimental results}

In order to establish a comparison between two types of APF filters, it was conducted some simulations with a prototype of the APF. Figure 4 represents the simulation schematic diagram of the photovoltaic system with linear load and shunt APF. In this case, the only source of harmonic in the system is represented by the photovoltaic inverter and, therefore, the APF will have to compensate selectively the harmonics injected from the photovoltaic system.

Given that the harmonic content depends and changes to the loading conditions of the photovoltaic system, several typical loading conditions have been considered to evaluate the flexibility and performance of the APF proposed, taking into account dynamic changes in the electrical parameters of the system. The APF must fit permanently to all operational conditions. These conditions are dynamics due to the changes in the climate variables that define the performance of the photovoltaic system. The loading conditions used are:

- Very low loading: Between $5 \%$ and $10 \%$ of the nominal power of the photovoltaic inverter. This case represents the most adverse condition of operation, because the harmonic content is the highest according to Fig. 2.

- Low loading: Between $10 \%$ and $20 \%$ of the nominal power of the photovoltaic inverter.

- $\quad$ Mid loading: Between $40 \%$ and $50 \%$ of the nominal power of the photovoltaic inverter.

The case of high loading has not been considered because, for this loading condition in the photovoltaic system, the harmonic content that the inverter injects is very low and negligible, as it is appreciated in Figure 3.
Table I. Cases considered for simulation

\begin{tabular}{|l|c|c|}
\hline $\begin{array}{l}\text { Loading conditions of the } \\
\text { photovoltaic inverter }\end{array}$ & Type of load & $\begin{array}{c}\text { Simulation } \\
\text { case }\end{array}$ \\
\hline Very low loading & Linear & Case I \\
\hline Very low loading & No Linear & Case II \\
\hline Low loading & Linear & Case III \\
\hline Medium loading & Linear & Case IV \\
\hline
\end{tabular}

Table I summarize each of the cases simulated with the APF proposed during the development of this work. The main purpose of these simulations is to verify that the filters inject into the PCC the necessary currents to compensate selectively the harmonic distortion of current that generates the photovoltaic inverter.

As previously mentioned, in the photovoltaic system the inverter is the main responsible of the harmonic injection to the network, therefore to reproduce the harmonic content that generates the photovoltaic system, in the simulations is enough to represent the inverter by means of the voltage source at fundamental frequency and harmonic current sources that changes its loading from the climatic conditions [11].

For the simulation was used a three-phase photovoltaic inverter of $500 \mathrm{~kW}$; technical data were obtained from the Inverters Catalogue of Schneider Electric.

The photovoltaic inverter has been simulated as a voltage source in parallel with current sources. The voltage source is responsible for supplying of the fundamental current and fixing the voltage level of the system; whereas, the current sources are responsible for generating the harmonic currents that, as a whole, represent the harmonic content injected by the inverter.

Four cases were simulated for both, APF with conventional control and APF with the selective control scheme. Results show that the APF with selective control presents better results than the other one for all cases.

\section{Case II}

It is shown the results for both APF's for case II (severe case). Figure 6 shows the harmonic content without APF filter.

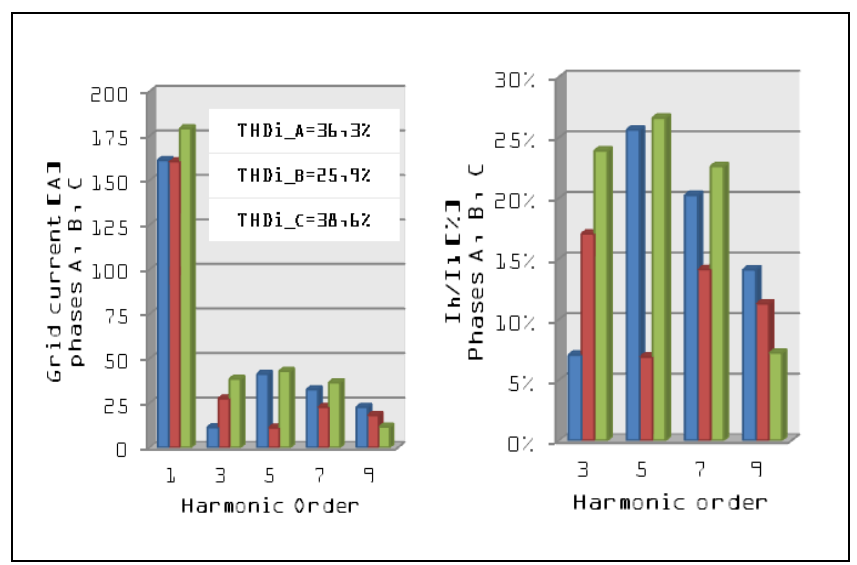

Fig. 6. Harmonic current in the network without APF with for Case II of the Table I 


\section{A. Results for APF with conventional control}

The results show that, the APF with conventional control does not compensate the current harmonic content from the inverter and the non linear load. The filter reduces the THDi form $38,6 \%$ to $32,4 \%$, this level is too high taken into account international standards where the limits are near to $5 \%$.

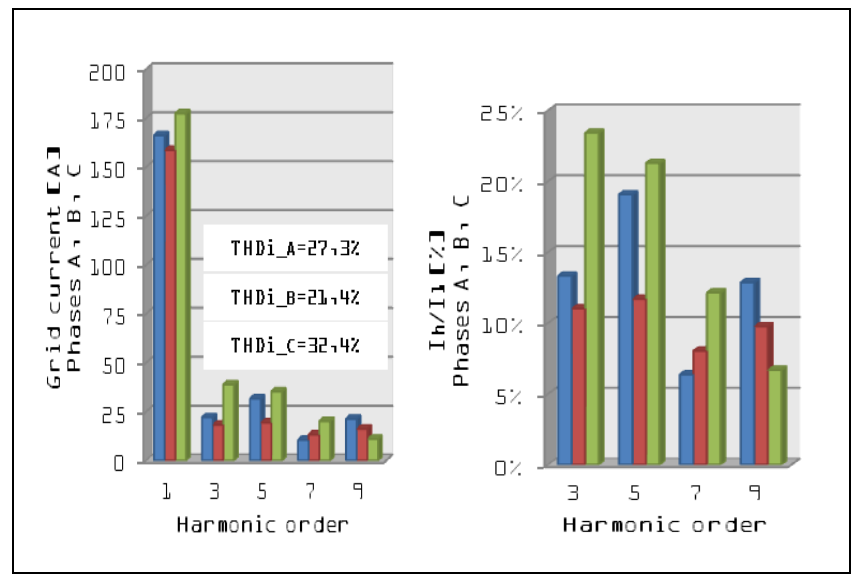

Fig. 7. Harmonic current in the network with APF with conventional controller for Case II of the Table I

\section{B. Results for APF with selective control.}

The results show that, for this case, the APF proposed compensates the harmonic currents individually, including those of high magnitude, and consequently, decreasing the $\mathrm{THD}_{\mathrm{i}}$ in the network (from 38,6\% to 5,6\%). These results indicate that the APF proposed is sufficiently selective to identify and to compensate those individual harmonics of high magnitude, as it is shown in Figure 8 where the harmonics 3, 5 and 7 were reduced at lower levels than $1 \%$.

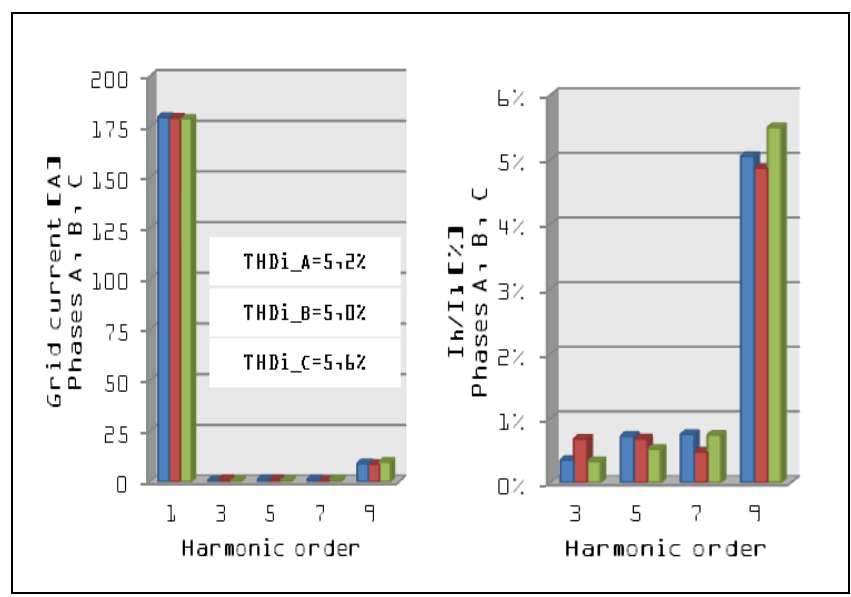

Fig. 8. Harmonic current in the network with APF with selective controller for Case II of the Table I

Now, it shows the result with APF with selective control in case III. The APF with conventional control are not shown because the performance is quite similar with case II. For more information please refer to [1].

\section{Case III}

The results show that in this case, the APF proposed compensates harmonic currents individually and, consequently, decreasing the $\mathrm{THD}_{\mathrm{i}}$ in the network (from
$9,9 \%$ to $0,7 \%)$.

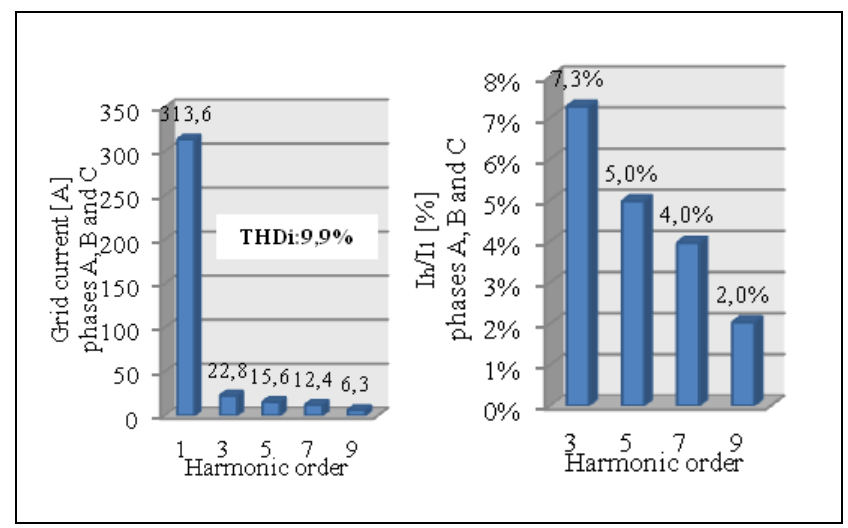

Fig. 11. Harmonic current in the network without APF with selective controller for Case III of the Table I

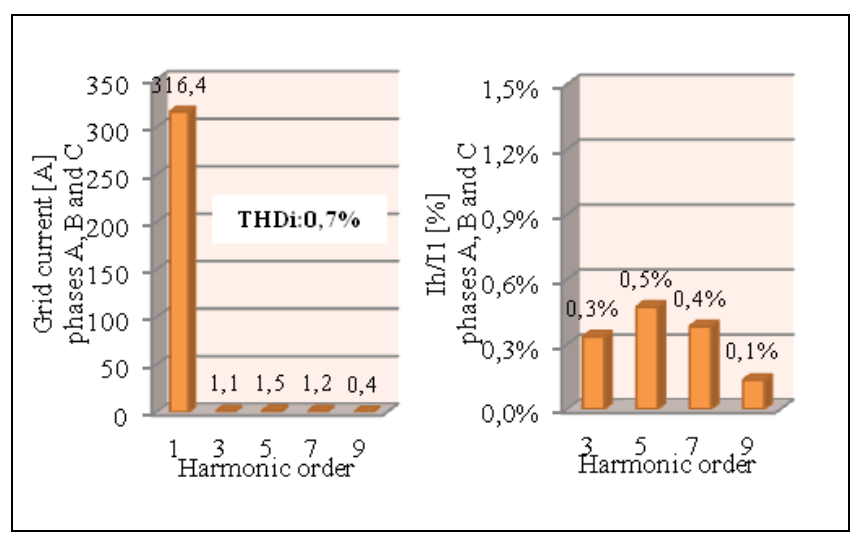

Fig. 12. Harmonic current in the network with APF with selective controller for Case III of the Table I

\section{Conclusions}

From this work, five fundamental conclusions can stand out:

- The harmonic current presented in the photovoltaic generation systems is mainly generated in the inverter device. This characteristic was modeled and tested in this work with two types of active filters, one of them conventional controller and the other one selective controller, in order to eliminate the harmonic content.

- Results show that the APF with selective controller reduces the harmonic content to standard values (around 5\%), whereas the APF with a conventional controller did not able to reduce them.

- The proposed APF with selective controller, demonstrates to be the sufficiently selective to identify and to compensate those individual harmonics of high magnitude, for example, the harmonics 3, 5 and 7 who were the highest according to the magnitude given in Figure 3 .

- The proposed APF with selective controller was able to reduce the individual harmonic and THDi from photovoltaic systems, according to international standard IEEE 519. 
- The results of this work are a novel proposal, since studies before to this, always have been interested in the compensation of harmonics arising from nonlinear loads but rarely from a voltage source, as in this case.

\section{Acknowledgement}

The first author wants to express his gratitude to Javier Roldán, who knowledge allowed to carry out this research and Ph.D. Omar Pinzón because much of this work is based in previous researches done by him. The authors express their gratitude to COLCIENCIAS and Universidad del Norte.

\section{References}

[1] Osorio, J. Filtro activo de potencia con esquema de control selectivo para la compensación de las componentes armónicas en los inversores fotovoltáicos de conexión a red. Master Thesis presented at the Universidad del Norte, Barranquilla, 2012.

[2] Chicco, G., Schlabbach, J., \& Spertino, F. (2005). Characterisation of the harmonic emission of grid-connected PV-systems. Proc. of IEEE Conference St. Petersburg PowerTech 2005 (66).

[3] Francis, B., \& Wonham, W. (1975). The internal model principle for linear multi-variable regulators. Applied Mathematics and Optimization (2), 170-194.

[4] Franklin, G. F., Powell, J. D., \& Workman, M. L. (1997). Digital Control of Dynamic Systems (3rd edition ed.). Addison-Wesley.

[5] García-Cerrada, A., Roncero-Sánchez, P., García-González, P., \& Feliu-Batlle, V. (2004). Detailed analysis of closedloop control of output-voltage harmonics in voltage-source inverters. IEE Proceedings on Electric Power Applications , 734-743.

[6] García-Gonzalez, P., \& García-Cerrada, A. (2000). Control system for a PWM based STATCOM. IEEE Transactions on Power Delivery, 1252-1257.

[7] González G., R., Jiménez G., H. R., \& Lagunas M., J. (Octubre-Diciembre de 2003). Sistemas fotovoltaicos conectados a la red. Boletín IIE , 5 .

[8] Mun, O. C. (1998). Dynamic simulation of electric machinery using Matlab. Prentice Hall.

[9] Pinzón Ardila, O. (2006). Aplicación de la eliminación selectiva de armónicos a filtros activos de potencia. Seminario Internacional de Ingeniería Electrónica UPB. Colombia.

[10] Pinzón Ardila, O. (2011). Compensación selectiva de armónicos con filtros activos de potencia. EAE.

[11] Schlabbach, J. (May de 2008). Harmonic current emission of photovoltaic installations under system conditions. Electricity Market, 2008. EEM 2008. 5th International Conference on European , 1-5. 